\title{
Paraplegia
}

\section{Osteonecrosis: An Overuse Injury of the Shoulder in Paraplegia: Case Report}

\author{
D. B. Barber, MD, N. G. Gall, MD \\ Department of Physical Medicine and Rehabilitation, University of Texas Health \\ Science Center at San Antonio, 7703 Floyd Curl Drive, San Antonio, Texas \\ 78284-7798, USA.
}

\begin{abstract}
Summary
The shoulder of the wheelchair dependent paraplegic is subject to overuse injury with subsequent pain. The major overuse syndromes observed include soft tissue injuries and secondary degenerative arthritis. This report presents a case in which bilateral osteonecrosis of the humeral heads was found to be the source of pain in the shoulders of an active paraplegic without any evidence of disease or medical treatment associated with the development of osteonecrosis. Osteonecrosis should be entertained in the differential diagnosis of overuse injuries of the shoulder in paraplegia.
\end{abstract}

Key words: Osteonecrosis; Paraplegia; Humerus; Shoulder

The shoulder of the wheelchair dependent paraplegic is the primary joint used for transfer and ambulation. As a consequence of providing these new functions in addition to 'ordinary use', the shoulder is subject to overuse injuries with resultant pain. The major syndromes observed from overuse include soft tissue injuries such as subdeltoid bursitis, bicipital tendonitis, and rotator cuff tear as well as secondary degenerative arthritis (Bayley et al., 1987; Gellman et al., 1988; Wylie and Chakera, 1988). In addition to soft tissue injury, Bayley et al., (1987) discovered osteonecrosis of the humeral head in 5 out of 31 paraplegics with shoulder pain. They were not able to determine whether these 5 patients had any predisposing disease or medical treatment associated with the development of osteonecrosis; however, 4 of the 5 patients admitted to habitual moderate to heavy intake of alcohol. Although the average time between spinal cord injury and the estimated time of onset of symptoms was 19 years, the development of osteonecrosis in those 5 patients cannot be attributed solely to chronic overuse with complete confidence. The following report, however, presents a case of bilateral osteonecrosis of the humeral heads in a paraplegic person without any evidence of disease or medical treatment associated with osteonecrosis, implicating overuse as the etiology. 


\section{Case report}

The patient is a 37 year old male with a 3 year history of T8 anterior cord syndrome secondary to a traumatic thoracic aortic aneurysm with compromise of flow to the anterior spinal artery. He subsequently achieved functional independence at a T8 level and was extremely active. He presented with a chief complaint of progressively worsening bilateral should pain of approximately 6 months duration. The pain was described as 'dull aching' in nature and was noted to be continuous in duration. It was associated with movement, particularly abduction against resistance and transfers. Pain was also present at rest. Infrequently he was awakened from sleep by this pain. He denied any antecedent trauma prior to the development of his symptoms.

Physical examination noted full active range of motion and no focal weakness about the shoulders bilaterally. Tenderness to palpation was appreciated at the glenohumeral joints bilaterally and the left acromioclavicular joint. Provocative tests (i.e. Drop test, Yergason's sign, etc.) for identification of rotator cuff tear, impingement syndrome, and other soft tissue injuries were negative.

Roentgenograms of the shoulders were obtained and revealed sclerosis in the subarticular bone of the head of the humeri with some alteration in the contour of the articular surfaces consistent with stage II osteonecrosis by the staging criteria of Ficat and Arlet (1980) adapted to the humerus (Figure). A 99m-Tc-MDP bone scan was obtained and displayed increased uptake in the humeral heads and in two ribs. Uptake in the ribs was felt to be secondary to old trauma; however, uptake in the humeri was felt to be consistent with chronic osteonecrosis at a stage of revascularisation and repair (Bonnarens et al., 1989).

An extensive review of the patient's medical records found him to be otherwise in a state of excellent health with no major medical conditions except recurrent ischial decubitus ulcers, no evidence of traumatic injury to his humeri at the time of his spinal cord injury, and no history of systemic corticosteroid use or steroid injections into his shoulder girdle. His social history was significant for modest but not habitual alcohol consumption.

After multidiscipline evaluation, a conservative treatment plan was developed in an attempt to prevent further progression of the osteonecrosis while allowing for an attempt at natural repair to take place. This treatment plan consisted of preserva-

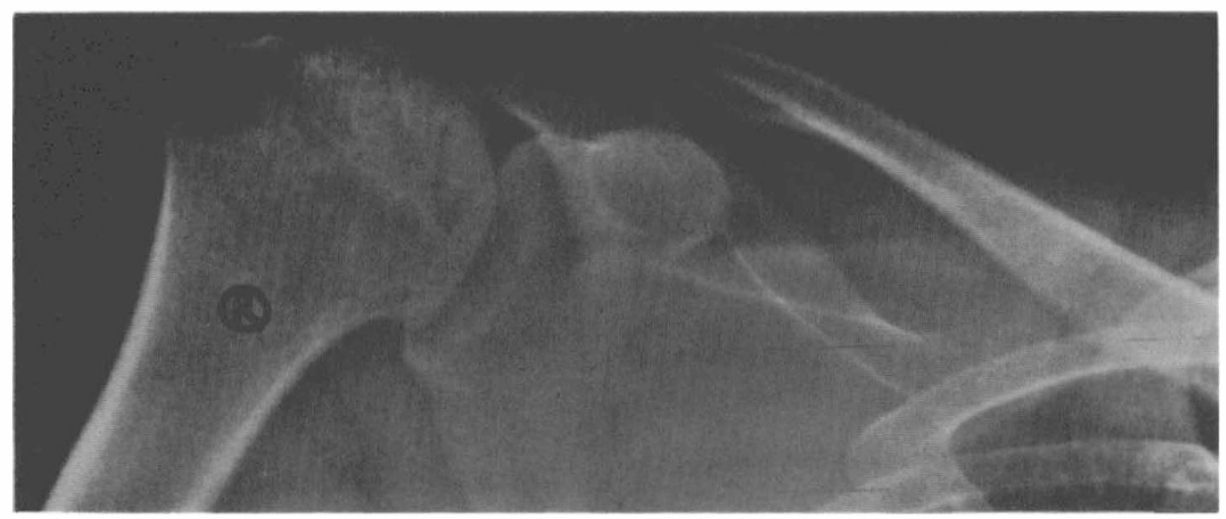

Figure X-ray of right shoulder with alteration of the surface of the humeral head. 
tion of motion through a program of pendulum exercises while avoiding abduction against resistance, symptomatic pain reduction through the use of local heat and nonsteroidal anti-inflammatory agents, and reduction of unnecessary shoulder work through conservation of activity (Cruess, 1985). The patient has been followed with serial roentgenograms and these have demonstrated no further progression of the osteonecrosis now approximately 18 months from the time of diagnosis. In addition, the patient has maintained functional independence and the pain has been controlled to within tolerable limits.

\section{Discussion}

It appears likely that the development of osteonecrosis is multifactorial in nature. As previously stated, numerous disease processes and their medical treatment have been associated with osteonecrosis (Zizic, 1985). However, up to $24 \%$ of cases in large series have been considered idiopathic (Zizic, 1985).

Certain sites, primarily the subchondral areas of long bones, are particularly susceptible to the development of osteonecrosis. In his series of patients with involvement of the humeral head secondary to corticosteroid use, Cruess (1985) identified the osteonecrotic defect in that portion of the humeral head that articulates with the glenoid at $90^{\circ}$ of abduction. This subchondral region is vulnerable due to a number of factors: (1) the lesion develops immediately below the load-bearing surface; (2) the blood supply is an endarterial system with little potential for development of collateral circulation in the event of injury; (3) there is prominent fatty marrow with narrower capillary circulation than hematopoietically active marrow; and (4) because of its rigidity, increase pressure within the bone can have profound effects on the microcirculation. The final common pathway often appears to be elevated bone marrow pressure producing increased resistance to blood flow with resultant ischemia and cellular damage (Zizic, 1988).

Although this case report would by definition be classified as idiopathic, we believe the mechanism which resulted in the development of osteonecrosis to be that of overuse of a joint called upon to assume demanding functions secondary to the patient's paraplegia. Bayley et al., (1987) measured the intra-articular pressure in the shoulder of 5 asymptomatic paraplegics during transfer. Intra-articular pressures were found to exceed mean arterial pressure by more than two and a half times. Through wheelchair propulsion, transfers, and the normal activities that the shoulder is called upon to perform, both the vascular supply and subchondral bone of the humeral head must surely receive repeated insults that cumulatively result in ischemia and damage. Thus, as demonstrated through this case report, osteonecrosis of the humeral head should be entertained in the differential diagnosis of overuse injuries of the shoulder in paraplegia.

\section{References}

Bayley JC, Cochran TC, SLEDGE CB 1987 The weight-bearing shoulder: The impingement syndrome in paraplegics. The fournal of Boint and Surgery 69A:676-678.

BonNaRENS F, Hernandez A, D'Ambrosio R 1985 Bone scintigraphic changes in osteonecrosis of the femoral head. Orthopedic Clinics of North America 16(4):697-703.

CRUESS RL 1985 Corticosteroid-induced osteonecrosis of the humeral head. Orthopedic Clinics of North America 16(4):789-796. 
FICAT P, ARLET J 1980 Ischemia and Necrosis of Bone. Williams \& Wilkins, Baltimore, p. 68 GELLMAN H, SIE I, WATERS RL 1988 Late complications of the weight-bearing upper extremity in the paraplegic patient. Clinical Orthopaedics and Related Research 233:132-135.

WYLIE EJ, CHAKERA TM 1988 Degenerative joint abnormalities in patients with paraplegia of duration greater than 20 years. Paraplegia 26:101-106.

ZiZIC TM, HUNGERFORD DS 1985 Avascular necrosis of bone. Textbook of Rheumatology, 2nd ed. WB Saunders, Philadephia, pp. 1689-1910.

ZIZIC TM 1988 Osteonecrosis. Primer on the Rheumatic Diseases, 9th ed. Arthritis Foundation, Atlanta, pp. 253-256. 